\title{
PHENOTYPIC MODULATION OF SMOOTH MUSCLE CELLS AND MATRIX METALLOPROTEINASES AS TARGETS FOR ATHEROSCLEROTIC PLAQUE STABILIZATION
}

\author{
George N. Chaldakov ${ }^{*}$, Maria D. Zhelyazkova-Savova ${ }^{2}$, Daniela Panayotova ${ }^{3}$, Marco Fiore ${ }^{4}$, and \\ Stanislav Yanev ${ }^{5}$ \\ ${ }^{1}$ Department of Anatomy and Cell Biology, Medical University, Varna, Bulgaria, \\ ${ }^{2}$ Department of Pharmacology and Clinical Pharmacology and Therapeutics, Medical University, \\ Varna, Bulgaria, \\ ${ }^{3}$ Department of Cardiac Surgery, St Marina University Hospital, Varna, Bulgaria, \\ ${ }^{4}$ Institute of Biochemistry and Cell Biology, Section of Neurobiology, National Research Council (CNR), \\ Rome, Italy, \\ ${ }^{5}$ Laboratory of Drug Toxicology, Institute of Neurobiology, Bulgarian Academy of Sciences, \\ Sofia, Bulgaria
}

Atherosclerosis and its complications, erosion and rupture of the plaque fibrous cap, lead to myocardial infarction and stroke, the main causes of mortality worldwide. In this setting, arterial smooth muscle cells (SMC) of the innermost media undergo phenotypic changes, a switch towards a secretory phenotype engaged in matrix proteins production. In its nature, this is a protective action that forms of a new arterial layer, the fibrous cap covering the plaque thrombogenic lipid core. The risk of plaque rupture is inversely correlated with the presence of secretory state SMC and collagen fibrils within the fibrous cap. Thus, fibrous cap remodeling appears to be the main determinant of plaque vulnerability. Herein, we focus on the potential role of (i) the transcription factors TCF21 and KLF-4 in SMC phenotypic modulation, (ii) the matrix protein secretion of SMC, and (iii) the activity of proteinases (MMP, ADAM, ADAMTS, furin, and the MMP inducer CD147) in this critical process. We argue that focusing on these basic pathways could contribute to the knowledge of fibrous cap stability that might be translated into clinical medicine.

Biomed Rev 2020; 31: 49-60

Keywords: atherosclerosis, fibrous cap, smooth muscle cells, secretion, collagen, matrix metalloproteinases, TCF21, KLF-4, furin, CD147

\footnotetext{
*Correspondence to: Dr George N. Chaldakov, Department of Anatomy and Cell Biology, Medical University, BG-9002 Varna, Bulgaria E-mail: chaldakov@yahoo.com; Mobile: +359 888679204
} 


\section{INTRODUCTION}

\section{Binary nature of cell life}

Since "the beginning, long time ago", life at cellular and molecular level is a binary (Yin-Yang), dynamic and holistic event aimed at the maintenance of a sanogenic phenotype of the homeostasis. Quite the contrary, dyshomeostasis is the cellular and molecular signature of a pathogenic phenotype (1).

For instance, the progression of the atherosclerotic plaque is, at least in part, a consequence of non-resolved inflammation due to an imbalance between the inflammatory lipid mediators leukotriene B4 and prostaglandin E2 (say Yin) and the resolving lipid mediators resolvin D2 (RvD2), maresin 1 (MaR1) and resolvin E1 (RvE1) (say Yang). In a therapeutic context, repetitive RvD2 and MaR1 delivery may prevent atheroprogression via a decrease in lymphocytes and macrophages accumulation along with increased fibrous cap thickness $(2,3)$.

Among others, an example of such a dual functionality appears to be the one that includes matrix metalloproteinases (MMP), a disintegrin and metalloproteinases (ADAM) and ADAM with thrombospondin motifs (ADAMTS), together with the endogenous tissue inhibitors of metalloproteinases (TIMP).

Herein, we focus on the potential role of (i) the transcription factors TCF 21 and KLF-4 in SMC phenotypic modulation, (ii) the matrix protein secretion of SMC, and (iii) the activity of proteinases (MMP, ADAM, ADAMTS, furin, and the MMP inducer CD147) in the remodeling of the fibrous cap towards its stabilization, that is, as an anti-atherosclerotic action. It is an updated and enlarged version of our 2019 Dance Round (2).

\section{FIBROUS CAP STRUCTURE AND FUNCTION}

In the 12th century, the French theologian Alain de Lille wrote Mille viae ducunt homines per saecula Roman (A thousand roads lead men forever to Rome). In the present context, this maxim may be expressed as "Many roads lead to (athe) roma". One of these is the vascular dyshomeostasis induced by multiple genetic, epigenetic and lifestyle factors.

The process of atherogenesis takes place mainly within the inner coat (tunica intima) (also adventitia and tunica adiposa) of the artery wall. In this setting, a vascular remodeling emerges as consisting of the phenotypically modulated medial smooth muscle cells (SMC) and adventitial fibroblasts toward matrix protein secretion; for mesenchymal stem cells, see (46). All these events aim at the formation of a protective layer of the injured artery wall, that is, the plaque fibrous cap.

Recent advances have shed light on the relationship between
SMC biology, inflammation, matrix proteinase activity and plaque vulnerability-stability. Plaque instability has been associated with erosion, ulceration or rupture of the fibrous cap. The thick fibrous cap of atherosclerotic plaques is composed of bundles of SMC and collagen fibers and few macrophages and lymphocytes. The thin fibrous cap contains many macrophages and lymphocytes, few SMC and less collagen fibrils, leaving the plaque prone to rupture and thrombosis (Table 1, Fig. 1).

\section{Table 1. Major features of unstable atherosclerotic plaque}

Thin fibrous cap $(<100$ micrometer)

Increased presence of macrophages and lymphocytes

Low presence of SMC and collagen fibers

Large lipid core ( $>30 \%$ of the total volume of plaque)

Presence of intra-plaque hemorrhage

Cap erosion and thrombus formation

Increased MMP/TIMP ratio

Increased expression of CD147, a MMP inducer

Altered expression of ADAM and ADAMTS

Increased expression of furin (PCSK3)

Decreased expression of transcription factor 21 (TCF21)

\section{REMODELING OF THE FIBROUS CAP}

Perhaps, every pathology, including atherosclerosis, emerges as a variation of response-to-injury (7). Tissue remodeling, the reorganization of existing tissue, is an essential phenomenon in this process (Fig. 2). Just to remind, the renowned Russell Ross's background in research was in skin biology, particularly, wound repair. Most probably, in consequence of that, he made notable contributions to the understanding of the pathogenesis of atherosclerosis. In 1973, he, together with John Glomset, formulated the response-to-injury hypothesis of atherosclerosis (7 and references therein).

In the context of atherosclerosis, vascular remodeling refers to a protective change in the artery wall due to the plaque growth. The SMC in a secretory state may "repair" atherosclerotic plaques (vascular wounds) by the formation of fibrous cap, which is rich in type I, II, and III fibrillar collagens, covering the plaque lipid core. The destruction of collagens, proteoglycans and other extracellular matrix (hereinafter, matrix) proteins of the fibrous cap, due to elevated MMP and reduced TIMP activity, is considered to precipitate plaque rupture and its clinical sequelae such as myocardial infarction and stroke. In short, in stable plaque the thrombogenic lipid core is protected from the circulating blood by a fibrous (collagenous) cap. 

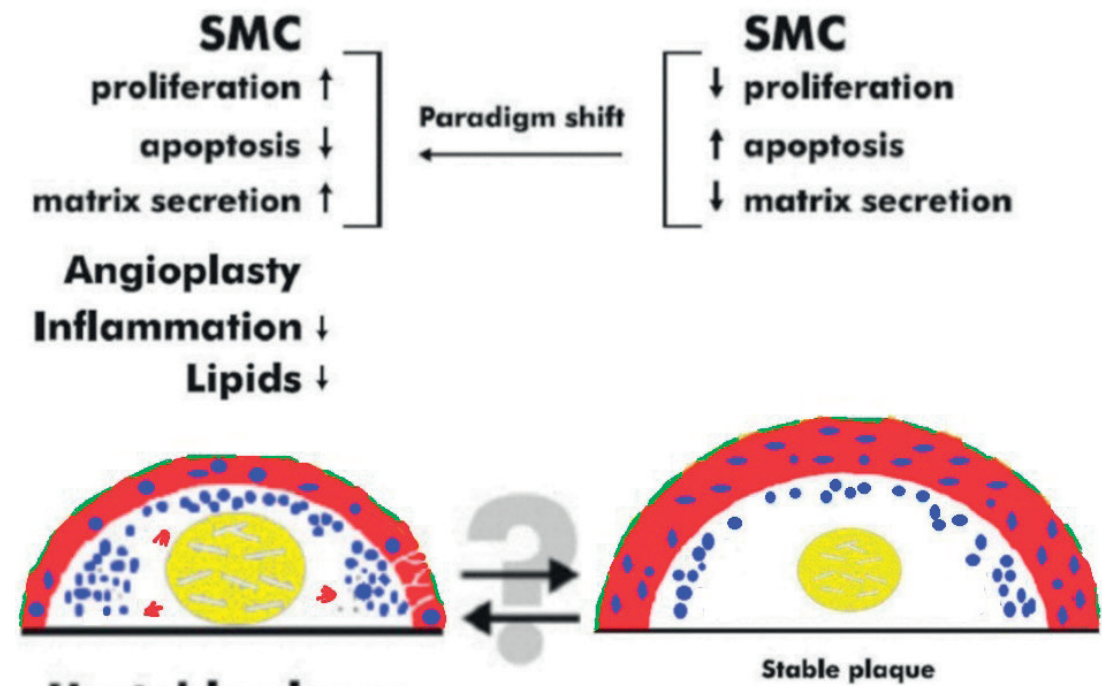

Unstable plaque

Figure 1. Upper part, an illustration of the paradigm shift in the process of atherogenesis (long arrow), SMC, smooth muscle cells. Lower part, both plaques are covered by a single layer of endothelial cells (green) and fibrous caps (red). The unstable plaque has a thin fibrous cap containing macrophages (blue granules) and few SMC (blue spindles), which may weaken the cap, leaving it vulnerable to rupture (white lines in cap's shoulder (at right). On the contrary, the stable plaque has a thick fibrous cap composed of many SMC (blue spindles) and few macrophages (blue granules). Within the plaque, the lipid core (yellow) is larger in unstable than in stable plaques, and macrophages and lymphocytes (blue granules) are more prevalent in unstable than in stable plaques. In the unstable plaque, three intraplaque hemorrhages (red) are also depicted. From (2).

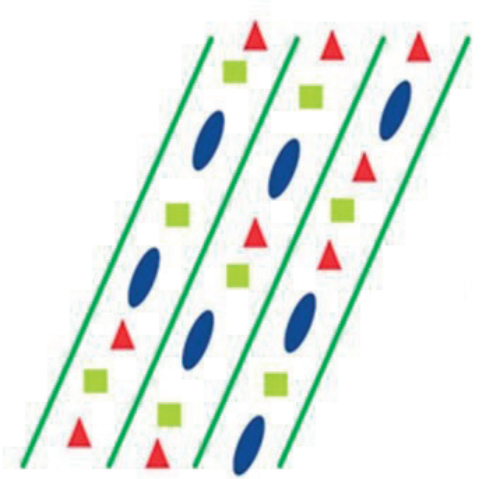

A. Health tissue

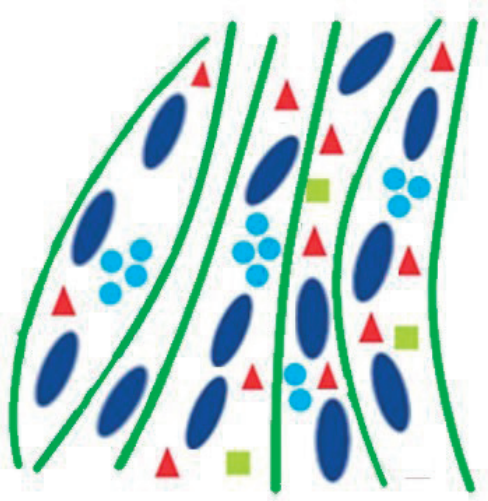

B. Physiological tissue remodeling

\section{Legend}

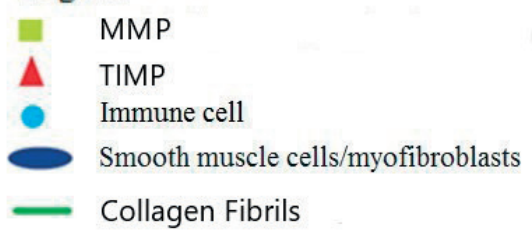

Figure 2. Schematic presentation of a physiological (protective) tissue remodeling in response-to-injury (B) as compared to a healthy tissue. Modified from (8). 


\section{Collagens}

There are 28 collagen types, numbered with roman numerals (I-XXVIII) in the human body. These constitute up to $30 \%$ of its total protein mass representing the most abundant proteins in mammals. In the human genome, 44 collagen genes code for polypeptide chains and are combined in diverse ways to form collagen fibrils. A characteristic feature of all fibrillar collagens is the presence of a tight right-handed triple helix composed of three polypeptides $\alpha$-chains, each of which contains one or more regions featured by the repeating amino acid motif $(\mathrm{Gly}-\mathrm{X}-\mathrm{Y})_{\mathrm{n}}$, with proline and 4-hydroxyproline amino acids often found at the $\mathrm{X}$ and $\mathrm{Y}$ positions, respectively. The triple helix motif can represent up to $96 \%$ of the collagen structure (for collagen type I) and to $<10 \%$ (collagen type XII) $(1,9)$.

\section{Lesson from collagen therapy of osteoarthritis}

Recent clinical investigations reported osteoarthritis improvement with nutraceuticals such as collagen hydrolysate and other collagen derivatives (10-12); for an experimental study, see (13). Weather this could be translated into the clinical setting of atherosclerotic plaque stabilization, remains to be investigated.

\section{Genes controlling SMC differentiation and phenotypic modulation}

Whereas the precise nature of the initiating event for atherosclerosis is not known, it is clear that the failure of the smooth muscle cell to maintain its normal differentiated phenotypic state becomes a key contributing factor in the progression of atherosclerotic disease (14).

The contractile properties of vascular SMC allow arteries and arterioles to regulate blood flow and pressure in response to acute demands of tissues. The resident SMC that constitute the majority of the artery wall also possess a remarkable structural and functional plasticity that allows them to undergo phenotypic modulation in both natural (15) and diseased (16-18) setting. While many cell types undergo irreversible terminal differentiation, differentiated (contractile state) SMC retain the ability to dedifferentiate to secretory state phenotype, a process known as phenotypic modulation (switch).

Arterial SMC are of pivotal significance for atherogenesis. In the classic response-to-injury hypothesis $(7,18)$, fully differentiated medial SMC express contractile marker genes and have limited proliferative capacity, while under disease conditions they downregulate contractile gene expression, secrete matrix molecules, proliferate and migrate into the neointimal space. It is generally accepted that SMC accumulation in the fibrous cap is monoclonal or oligoclonal, and this is consistent with the existence of a resident arterial subpopulation of SMC precursor cells that contribute to healing in the response to disease (19).

While the risk of plaque rupture appears to be inversely correlated with the number of SMC in the fibrous cap, there is limited understanding of their in vivo origin, and the molecular pathways that regulate their expansion and terminal phenotype determination. In this context, recent genome wide association studies have identified a number of genes that contribute to the risk for coronary atherosclerosis. It was demonstrated that upregulation of transcription factor 21 (Tcf21) gene for the transcription factor TCF21 (also known as capsulin and epicardin) plays a pivotal role in the development of epicardial progenitor cells that give rise to coronary artery SMC. Thus, TCF21 may have a role in the control of SMC precursor cells that migrate into vascular lesions and contribute to the fibrous cap in a way that stabilizes it and prevent heart attacks (Fig. 3; 17). Likewise, Wirka et al (20) provided multiple lines of evidence that (i) TCF21 promotes SMC transformation into a fibroblast-like phenotype ("fibromyocyte"), (ii) the loss of TCF21 inhibits this phenotypic modulation, thus, (iii) TCF21 expression is causally associated with reduced risk of coronary atherosclerosis. In brief, TCF21 up-regulation exerts its protective effect by promoting the accumulation of fibromyocytes into the fibrous cap. Meanwhile, the so-called "fibromyocytes" are, in fact, secretory state $\operatorname{SMC}(15,23)$

In the same "artery", recent studies indicate that Krüppellike factor 4 (KLF4) is an evolutionarily conserved zinc fingercontaining transcription factor, a member of the Krüppel-like factor family that regulates various cell processes, including the induction of pluripotent stem cells. KLF4-dependent phenotypic modulation of SMC is of a critical significance in the process of atherogenesis (21).

\section{Boosting the secretory pathways of SMC aiming at fibrous cap stabilization}

Or, how the foe becomes a friend in the fight against the atherosclerotic plaque (22). According to George Palade's classical concept and Gunter Blobel's signal hypothesis (23), the protein secretory pathway comprises several intracellular steps including synthesis, post-translational modifications, sorting, targeting, storage (in case of a regulated versus a constitutive secretion) and, finally, exocytosis (Fig. 4, 5). Non rough endoplasmic reticulum (RER)-Golgi complex pathway, including exosomes and ectosomes, should also be considered. Both secretory pathways might be targets for the discovery of 
Figure 3. TCF21 (transcription factor 21) in Vascular Disease Network built with differential gene expression data from siTCF21 RNA-Seq studies in HCASMC (human coronary artery smooth muscle cells). Interaction of the network nodes identified through enrichment of differentially expressed genes in functionally annotated categories was visualized with Cytoscape. Node color was mapped to log fold change with green representing genes that are downregulated along with TCF 21 and red representing genes that are upregulated, node size was mapped to absolute expression value in control cells, and font size to enrichment $Q$-value. Edges contain arrows to indicate the interection and are colored to distinguish types of interactions. Green edges represent functional interaction (proteinprotein binding, protein modification, molecular cleavage, phosphorylation, and protein-DNA interactions); magenta edges represent gene expression (expression and transcription) relationships; red edges represent activation; and

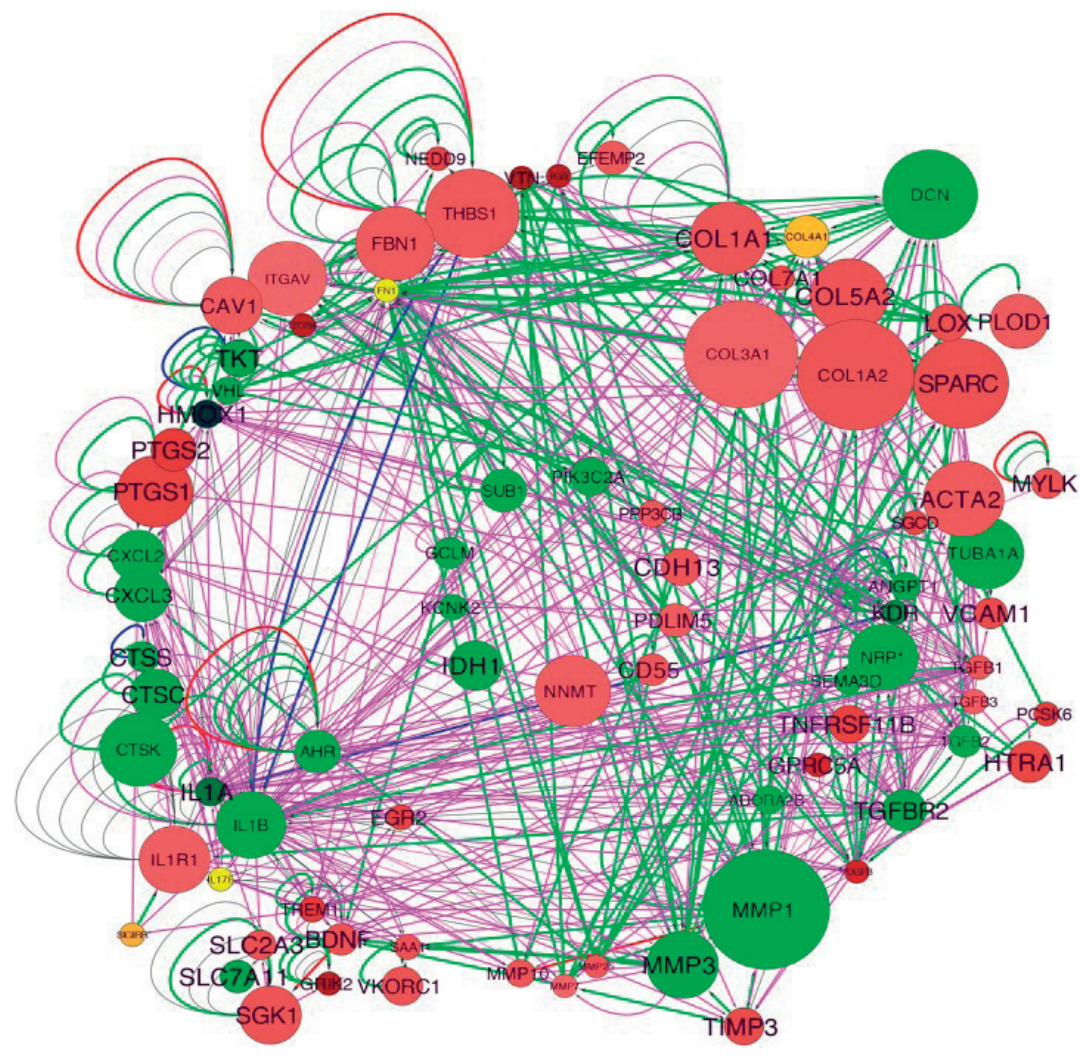
blue edges inhibition. From (17).

A. CELL
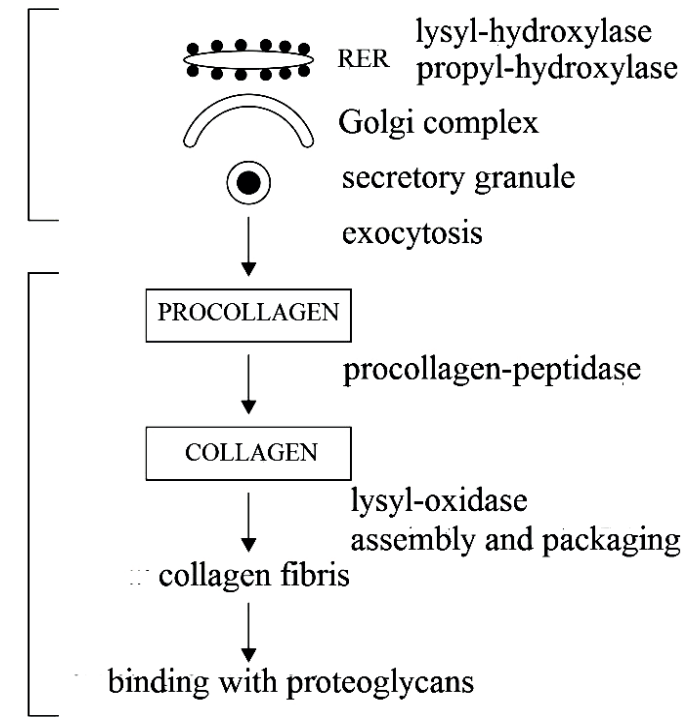

Figure 4. A schematic illustration of intra- and extracellular secretory pathways of procollagen to collagen biosynthesis. From (1).

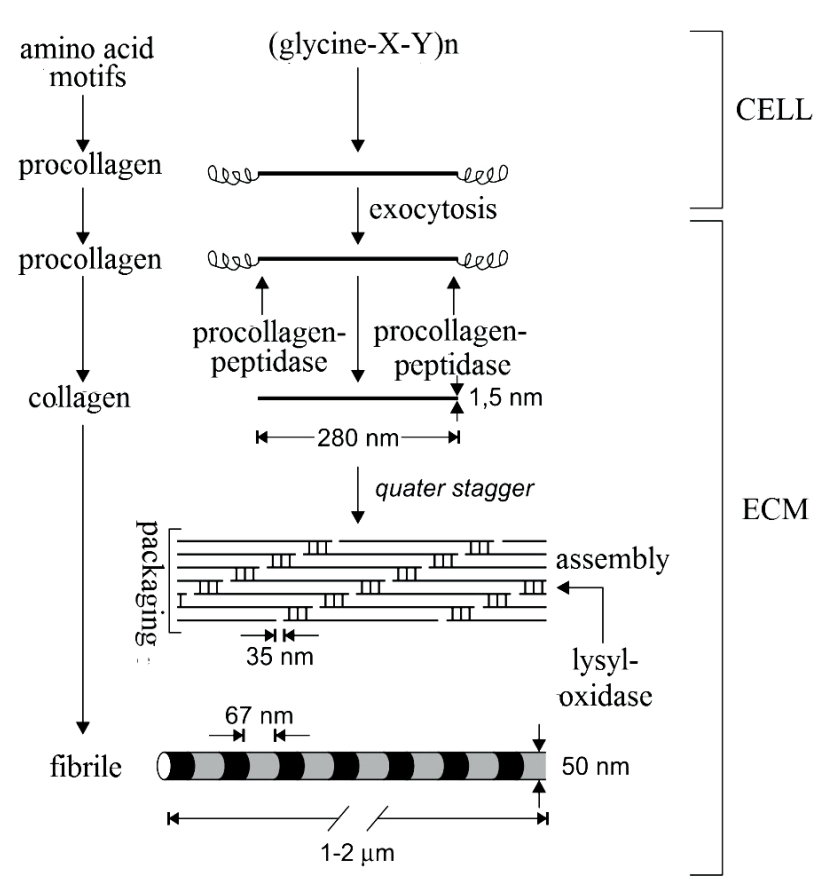

Figure 5. A more detailed illustration of intra- and extracellular secretory pathways of procollagen to collagen biosynthesis and packaging. From (1). 
new therapeutics for cardiovascular disease (24).

Accordingly, we should focus in-depth on Palade-Blobel's general theory of cell protein secretion to explore its pharmacological potentials for new therapies in atherosclerosis. Dissecting the matrix molecule secretion by microtubule-disassembling agents (e.g., colchicine, nocodazole, alike), microtubule-stabilizers (taxol, the drug paclitaxel), microtubule-based motor proteins (kinesin, dynein), or by Brefeldin A, an inhibitor of RER-Golgi complex trafficking, may provide important pharmacological information $(24,25)$. Further studies on SMC's matrix protein secretory pathways are pressingly required.

\section{MMP family of proteinases}

The MMP family of proteinases (also termed metzincins) consists of 26 human zinc-dependent endopeptidases (containing a zinc and methionine in their catalytic domain, hence, the name metzincins). These are MMP, ADAM and ADAMTS.

MMP are commonly classified on the basis of their substrates into collagenases (MMP-1, MMP-8, MM-13), gelatinases (MMP-2, MMP-9), stromelysins (MMP-3, MMP10, MMP-11), matrilysins (MMP-7), membrane-type (MTMMP-1-6, also known as MMP-14, 15, 16, 17, 24, 25) and miscellaneous (MMP-12, 19, 21, 23, 28). MMP are secreted by many cells including fibroblasts, vascular SMC, and leukocytes and have been localized in different areas of the atherosclerotic plaque, likely being involved in different, but also frequently overlapping, roles related to plaque remodeling and hence, stability-vulnerability status (26). MMP are often secreted in an inactive proMMP form, which is cleaved to the active form by various proteinases including other MMP. In addition to degrading different martix proteins, MMP also influence SMC migration and proliferation (26-28).

MMP play a critical role in various processes of vascular biology (Fig. 6). Alterations in specific MMP could influence arterial remodeling and lead to cardiovascular pathologies (Fig. 7).

Altogether, MMP appear to play dual roles in the pathobiology of atherosclerosis. The findings indicate that some MMP (MMP-2, MMP-3 and MMP-9) facilitate vascular SMC growth and therefore promote fibrous cap integrity, while other MMP (MMP-1, MMP-7, MMP-8, MMP-12, MMP-13 and MMP-14) lead to the loss of SMC and matrix proteins within the fibrous cap, thus contributing to its instability (27). Nevertheless, a clear differentiation in this respect cannot be made due to the complexity of interaction and the discordant results concerning different MMP, e.g. MMP-9, from experimental studies and clinical observations (29).

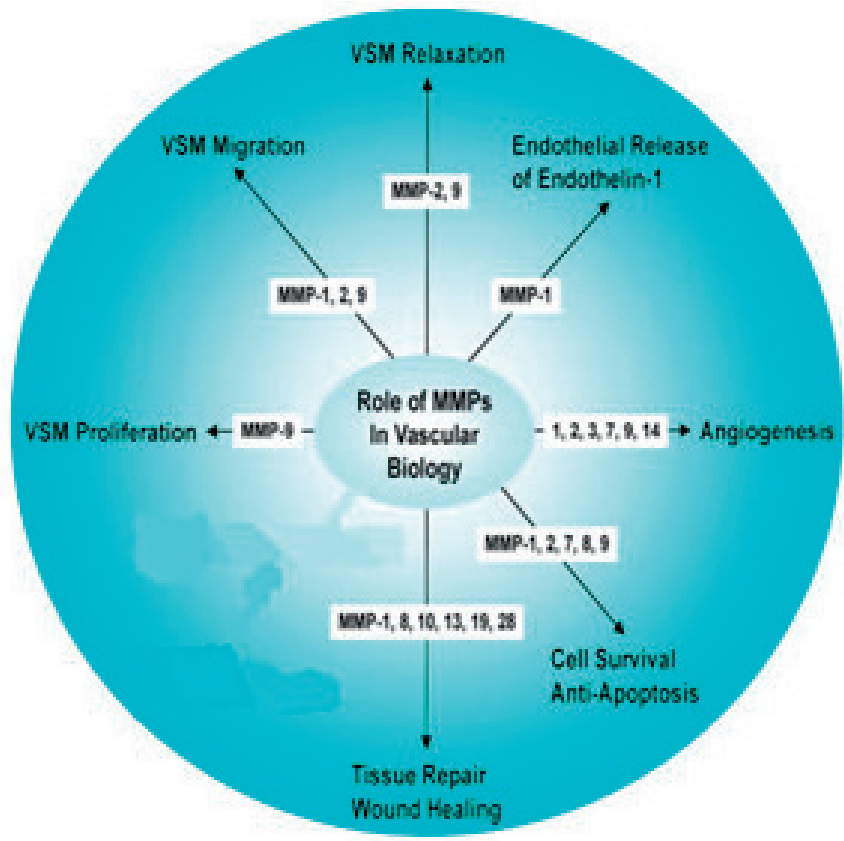

Figure 6. Examples of MMP roles in vasular biology. Modified from (28).

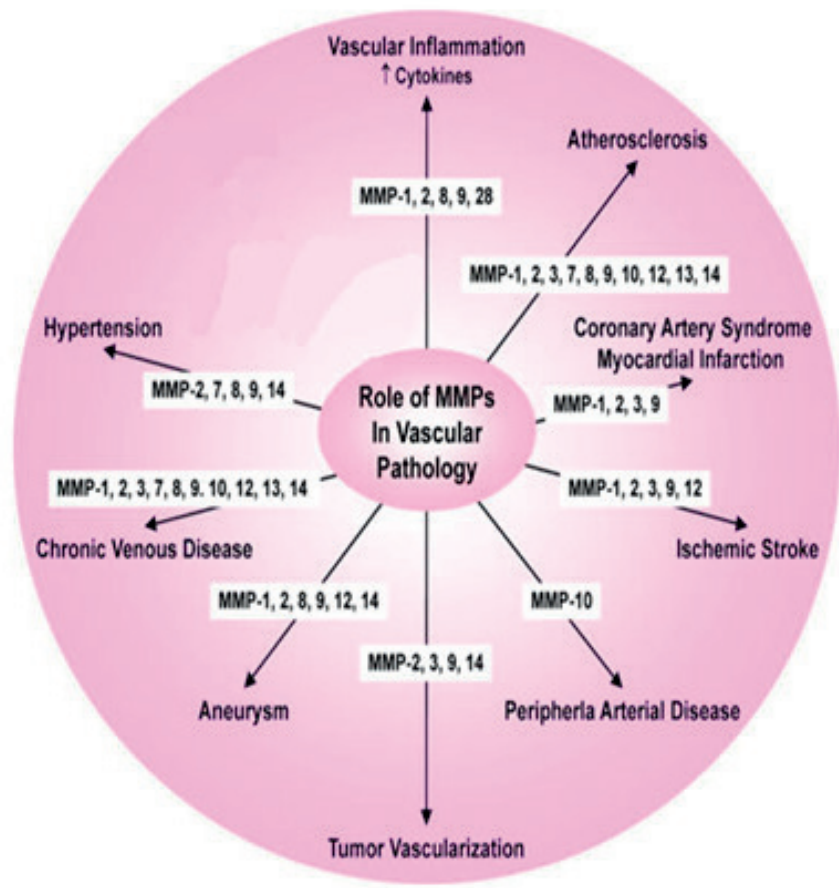

Figure 7. Examples of MMP roles in vascular pathology. Modified from (28). 


\section{MMP/TIMP ratio: biomarker and therapeutic target for atherosclerosis}

The tissue inhibitors of MMP (TIMP) play a key homeostatic role in regulating the activity of MMP and ADAMS, and as such they are commonly increased where MMP activity is prevalent. However, while stimulation of vascular SMC or macrophages with pro-inflammatory molecules induces the expression of multiple MMP, a concomitant increase in TIMP1 and TIMP-2 expression is not observed.

Generally, the destructive activity of MMP is tightly regulated by TIMP, the mechanism of action of which is dependent on their ability to chelate the zinc at the active site of MMP. In effect, MMP and TIMP harbour the potential to moderate all processes of atherogenesis - from formation, development and progression to plaque instability and thrombosis. Thus, the MMP/TIMP ratios might serve as biomarkers and therapeutic targets for atherosclerosis and other cardiovascular diseases. Circulating MMP-2 and MMP-9 (those that can be determined in the blood) may serve for the identification of patients who could profit from particular therapeutic intervention via inhibition of MMP pathway (26, 30-32).

\section{ADAM}

Among this family of proteinases, ADAM9, ADAM10, ADAM15, ADAM17 and ADAM33 have all been reported to be present within human atherosclerotic plaques at increased levels compared to healthy arteries. ADAM33 is preferentially present within plaque SMC. Genetic studies have also provided associative evidence for ADAM8, ADAM17, and ADAM33 expression in atherosclerosis. ADAM12 (meltrin12) was reported to be involved in the development of obesity (33).

\section{ADAMTS}

These proteases have also been implicated in atherosclerosis due to their ability to cleave proteoglycans such as versican, biglycan and aggrecan (34). Within human atherosclerotic lesions, ADAMTS1 is expressed mostly by SMC, and stimulate their migration, hence ADAMTS1 may favour plaque stability, however, the bulk of evidence suggests that ADAMTS1 may play a detrimental role in the etiology of atherosclerosis (35). On the other hand, ADAMTS4 and ADAMTS8 are prominent in the macrophage-rich regions and their expression increases during lesion progression. Loss of ADAMTS4 reduces atherosclerosis and enhances plaque stability in ApoE KO mice. Moreover, elevated circulating levels of ADAMTS4 have been reported in patients with coronary artery disease. Serum levels of ADAMTS4 have been associated with increased plaque vulnerability in patients with carotid artery stenosis (36). A protective role in atherosclerosis has been ascribed to ADAMTS5 as its expression was found depleted in atherosclerotic aortas, leading to the accumulation of proteoglycans such as versican and biglycan, which may favour lipid retention and macrophages. The risk of acute aortic dissection was independently associated with decreased plasma levels of ADAMTS5 (37).

\section{FACTORS INVOLVED IN PLAQUE STABILIZATION}

Possible major ways for the plaque stabilization are: (i) making plaque's cap more populated with secretory state SMC to be more fibrous, (ii) attenuating inflammation and oxidative stress, and (iii) reducing plaque lipid content, the first way being in the scope of present review.

\section{MMP inhibitors}

Mechanistically, since MMP may favor the degradation of matrix collagens and proteoglycans, the decrease of MMP activity may have a stabilizing action on the fibrous cap (26, 38-42). Different approaches have been tried to reach evidence, mostly experimental, but also clinical, in favor of the protective significance of inhibiting MMP.

\section{Doxycycline}

Doxycycline, an MMP inhibitor, significantly delays thoracic aorta aneurysm rupture in Marfan syndrome-like mice (43, 44). However, in apolipoprotein E-knockout mice, unfavorable results have been observed with doxycycline. Similarly, two independent placebo-controlled pilot clinical studies in patients with atherosclerosis have been disappointing, as doxycycline, given in sub-antibacterial doses, failed to produce any beneficial effect on the plaque phenotype or clinical outcomes. These unsatisfactory results have been attributed to the broad spectrum MMP inhibitory activity of doxycycline, supporting the notion that MMP play divergent roles during disease progression $(26,27)$.

\section{Macrolides}

Other antibiotics that have shown some experimental promise as MMP inhibitors are macrolides. The highest activity has been observed with clarithromycin and the other 14-member ring macrolides (erythromycin, roxithromycin). The initial interest to these drugs has been triggered by their anti-inflammatory activity and the hypothesis that atherosclerosis might have bacterial etiology. Clinical trials have yielded conflicting results. Lately, the potential of clarithromycin to inhibit MMP 
(MMP-9 particularly) and possibly to enhance TIMP-1, has attracted scientific interest. However, the concern of long-term safety associated with bacterial resistance, limits the clinical testing and thus new 14-member ring derivatives, devoid of antibacterial activity, are being currently sought to confirm the cardiovascular benefits of macrolides (45).

\section{Furin inhibitors}

Furin (Proprotein Convertase Subtilisin/Kexin Type 3; PCSK3) is a member of the proprotein convertase family that cleave multiple secretory protein precursors at specific single or paired basic amino acids. Activation of the membranetethered (MT) 1-MMP depends on furin. (Furin knockout mice die at embryonic day 11, due to cardiac ventral closure defects and hemodynamic insufficiency. However, furin ${ }^{+/-}$mice are viable, and appear relatively normal, suggesting that $\sim 50 \%$ of furin is sufficient to perform most of its critical functions.)

As a proprotein convertase, furin promotes the conversion of Pro-MMP2 to MMP2 via MT-1 MMP activation. Furin has been implicated in the pathogenesis of several diseases such as cancer, osteoarthritis, and atherosclerosis. Systemic inhibition of furin prevents atherosclerosis progression in mice, in part through the down-regulation of MMP2 activity. This may lead to increased plaque stability, thus representing a new therapeutic option for atherosclerosis (46).

Of note, the identification of the proprotein convertase subtilisin/kexin type 9 (PCSK9) as the third gene causing familial hypercholesterolemia and understanding its complex biology has led to the discovery of a novel class of therapeutic agents. PCSK9 is a critical regulator of LDL cholesterol metabolism, with an established role in atherosclerosis. The accepted mechanism at present for PCSK9's role in LDL metabolism and atherosclerosis is directly related to its role in the LDL receptor (LDLR) pathway. PCSK9 binds to LDLR at the plasma membrane and targets them to lysosomes for degradation (47). Interestingly, MMP2 overexpression in liver cells has been shown to prevent LDLR degradation induced by PCSK9, thereby showing a protective potential against atherosclerosis (48). A randomized open label clinical trial (NCT03552432) is currently being initiated at Kobi University to investigate the efficacy of alirocumab on plaque vulnerability in patients with coronary artery disease treated with a statin. Among the many secondary outcomes in this prospective study, the authors will also look for the dynamic changes of MMP-2 and MMP-9 during a 9-months therapy period.

Among the MMP inhibitors, the phosphinic peptide
RXP470.1, a potent and selective murine MMP-12 inhibitor, has been reported to reduce atherosclerotic plaque progression in apolipoprotein E-knockout mice (49).

\section{CD147 (basigin)}

CD147, also known as basigin, is an extracellular matrix metalloproteinase inducer (EMMPRIN), a transmembrane glycoprotein that belongs to the immunoglobulin superfamily. It acts as the stimulator of MMP and its expression is upregulated during various inflammatory processes, including atherosclerosis where it may contribute to plaque instability by inducing MMP expression. Thus, inhibition of CD147 may potentially have beneficial effects in the therapy of inflammatory disorders, including atherosclerotic cardiovascular disease. CD147-blocking antibodies, antagonistic peptides, and siRNA have been used in animal models to reduce tissue inflammation (50). A humanized IgG2 monoclonal antibody against CD147, meplazumab, has been developed as an antimalarial drug, but has been also shown to inhibit SARS-CoV-2 internalization and virus-induced inflammation since $\mathrm{CD} 147$ was identified as an additional entry receptor to infect host cell. A phase 2/3 randomized controlled double-blind clinical trial has been recently launched (NCT04586153) to evaluate the efficacy and safety of meplazumab in hospitalized adult patients with COVID-19, based on the encouraging results from a smaller observational study (51). It is also reasonable to expect promising results from mepalzumab in atherosclerosis therapy.

A number of drugs currently utilized in the treatment of atherosclerosis appear to exert some activity related to the role of MMP and their regulators.

\section{Renin-angiotensin aldosterone system}

The renin-angiotensin aldosterone system (RAAS) is a wellknown player in the cardiovascular remodeling. Angiotensin II (ATII) has been found to activate MMT-2 in young rats, and to mimic age-related carotid arterial remodeling (52). ATII was also shown to induce the expression of MMP-9 in cultured rat SMC, while losartan was able to inhibit MPP-9 production parallel to increasing TIMP-1, thus leading to reduced MPP-9/TIMP-1 ratio and providing mechanisms of preventing atherosclerosis (53). Experimental data show that aldosterone induces MPP-9 mRNA in human polymorphonuclear cells and spironolactone decreases its plasma levels; canrenone also significantly reduces MPP-2 and MPP-9 concentrations in patients with metabolic syndrome. The effects of these drugs on MPP can be considered not only directly related to RAAS 
inhibition, but also to their antioxidant and anti-inflammatory actions, given that among factors known to induce MMP expression and activation, are increased reactive oxygen species production and inflammation.

Dihydropyridine calcium channel blockers are thought to inhibit MPP-2 and MPP-9 through the same mechanisms (54). Statins

Statins, known for their multiple cholesterol-independent actions responsible for the plaque stabilization, also have been found to exert effects on MMP. Interestingly, differences were found in their potential to reduce MMP-9 and MMP-2, depending on the pharmacokinetic profile of the statins studied (55).

\section{PPAR-gamma agonists}

The thiazolidinedione drugs acting as PPAR-gamma agonist are recognized as antidiabetic medications reducing insulin resistance, but in terms of their cardiovascular safety, their benefits are balanced against potential harms. Still, both rosiglitazone (56) and pioglitazone (57) have been associated with reducing MMP that could account for their potential antiatherogenic effects.

A list of plaque stabilizers is shown in Table 2.

Table 2. A selected list of potential stabilizers of the atherosclerotic plaque

- Inhibitors of MMP

$\checkmark$ Doxycycline, a non-selective inhibitor

$\checkmark$ Macrolides (Clarithromycin, Erythromycin, Roxithromycin*)

$\checkmark$ RAAS inhibitors (ARBs, e.g., losartan; aldosterone antagonists e.g., spironolactone; renin inhibitors)

$\checkmark$ Dihydropyridine calcium channel blockers

$\checkmark$ Statins

$\checkmark$ PPAR gamma agonists

$\checkmark$ RXP470.1, an MMP-12 inhibitor

$\checkmark$ Furin (PCSK-3) inhibitors

$\checkmark$ PCSK-9 inhibitors (Evolocumab, Alirocumab ?)

$\checkmark$ CD147 inhibitors (Meplazumab ?)

- Activators of TIMP

- Modulators of ADAM and ADAMTM expression

- Stimulators of SMC proliferation

- Stimulators of SMC matrix protein secretion

- Inhibitors of SMC apoptosis

- Stimulators of transcription factor 21 (Tcf21) gene expression

- Transforming growth factor (TGF)- $\beta$ receptor antagonists**

- Exosomes***

- Inhibitors of MicroRNA-181b (miR-181b)

- Colchicine $e^{* * * * *}$

- Lipid mediators (resolvin D2, resolvin E1, maresin)*******

- Collagen hydrolysate (?)

${ }^{*}(58),{ }^{* *}(59),{ }^{* * *}(60),{ }^{* * * * *}(61),{ }^{* * * * * *}(62),{ }^{* * * * * *}(3)$

\section{CONCLUSION}

Unlike other cell types, the arterial SMC, also fibroblasts, are unique in their differentiation-dedifferentiation (phenotypic modulation) plasticity - from secretory, proliferative and migratory to contractile phenotype $(15-17,20)$. Hence, the topic of a recent paper "TCF21: Flipping the phenotypic switch in SMC" (63, see also 64) is very appropriate. Indeed, to move forth and back depending on changing environment during their life span is an unique behavior of these talented (7) cells.

Recently, the proof of a long standing hypothesis of a chronic inflammatory nature of atherosclerosis is becoming increasingly appreciated $(3,7,18,65,66)$. The present review aimed to explore that from another viewpoint, namely, the potential association of SMC phenotypic modulation, activities of MMP and atherosclerosis.

There is a general consensus that the majority of coronary syndromes are the result of rupture of unstable plaques and associated thrombotic events. Plaque instability is strongly associated with disruption of the fibrous cap, an atheroprotective arterial layer. These data are interpreted as providing evidence that plaques which contain a high ratio of both MMP/TIMP and macrophages/SMC are less stable, particularly those that have a thin fibrous cap composed primarily of contractile state of SMC.

Taken together, the results provide evidence that (i) the control on SMC phenotypic modulation, and (ii) the balance of activities of matrix proteinases harbour the potential to moderate all processes of atherogenesis - from formation, development and progression to plaque stability-instability. Hence, targeting at promoting beneficial changes in SMC phenotype and MMP/TIMP ratio may be viable therapeutic means for atherosclerosis and its clinical complications.

\section{COMPETING INTERESTS}

The authors have declared that no competing interests exist.

\section{ACKNOWLEDGMENTS}

The authors pay their tribute to Professor Delcho Zhelyazkov, the scientific father of one of them (GNC).

\section{REFERENCES}

1. Chaldakov GN. Principles of Cell and Tissue Biology. Textbook for medical students and all curious minds in biomedicine, 2021 (in press).

2. Yanev S, Zhelyazkova-Savova M, Chaldakov G. The fibrous cap: a promising target in the pharmacotherapy of atherosclerosis. Biomed Rev 2019; 30: 136-141. [DOI: 
10.14748/bmr.v30.6394]

3. Fredman G, Tabas I. Boosting Inflammation Resolution in Atherosclerosis: The Next Frontier for Therapy. Am J Pathol 2017; 187(6): 1211-1221. [DOI: 10.1016/j. ajpath.2017.01.018]

4. Li F, Guo X, Chen SY. Function and Therapeutic Potential of Mesenchymal Stem Cells in Atherosclerosis. Front Cardiovasc Med 2017; 4: 32. [DOI: 10.3389/ fcvm.2017.00032]

5. Yu H, Stoneman V, Clarke M, Figg N, Xin H-B, Kotlikoff $\mathrm{M}$, et al. Bone marrow-derived smooth muscle-like cells are infrequent in advanced primary atherosclerotic plaques but promote atherosclerosis. Arteriosc Thromb Vasc Biol 2011; 31(6): 1291-1299. [DOI: 10.1161/ATVBAHA.110.218578]

6. Bentzon JF, Falk E. Circulating smooth muscle progenitor cells in atherosclerosis and plaque rupture: Current perspective and methods of analysis. Vasc Pharmacol 2010; 52(1): 11-20. [DOI: 10.1016/j.vph.2009.11.005]

7. Ross R. Atherosclerosis - an inflammatory disease. $N$ Engl J Med 1999; 340(2): 115-126. [DOI: 10.1056/ NEJM199901143400207]

8. Chaldakov GN. KISS (Keep In Short and Simple) for the cell biology of fibroproliferative diseases. InSpiro 2019; 4(52): 1-12. In Bulgarian.

9. Bourgot I, Primac I, Louis T, Noël A, Maquoi E. Reciprocal Interplay Between Fibrillar Collagens and CollagenBinding Integrins: Implications in Cancer Progression and Metastasis. Front Oncol 2020; 10: 1488. [DOI: 10.3389/ fonc.2020.01488]

10. Bello AE, Oesser S. Collagen hydrolysate for the treatment of osteoarthritis and other joint disorders: a review of the literature. Curr Med Res Opin 2006; 22(11): 2221-2232. [DOI: 10.1185/030079906x148373]

11. Crowley DC, Lau FC, Sharma P, Evans M, Guthrie N, Bagchi M, et al. Safety and efficacy of undenatured type II collagen in the treatment of osteoarthritis of the knee: a clinical trial. Int J Med Sci 2009; 6(6): 312-321. [DOI: 10.7150/ijms.6.312]

12. Lugo JP, Saiyed ZM, Lane NE. Efficacy and tolerability of an undenatured type II collagen supplement in modulating knee osteoarthritis symptoms: a multicenter randomized, double-blind, placebo-controlled study. Nutr J 2016; 15 : 14. [DOI: 10.1186/s12937-016-0130-8]

13. Zhang Y, Kouguchi T, Shimizu K, Sato M, Takahata Y, Morimatsu F. Chicken collagen hydrolysate reduces proinflammatory cytokine production in C57BL/6.KORApoEshl mice. J Nutr Sci Vitaminol (Tokyo) 2010; 56(3): 208-210. [DOI: 10.3177/jnsv.56.208]

14. Owens GK: Role of alterations in the differentiated state of smooth muscle cell in atherogenesis. In: Fuster V, Ross R, Topol EJ, eds. Atherosclerosis and Coronary Artery Disease. Volume 1. Philadelphia - New York: LippincottRaven, 1996; 401-420.

15. Chaldakov GN, Vankov VN. Morphological aspects of secretion in the arterial smooth muscle cell, with special reference to the Golgi complex and microtubular cytoskeleton. Atherosclerosis 1986; 61(3): 175-192. [DOI: 10.1016/0021-9150(86)90137-1]

16. Gomez D, Owens GK. Smooth muscle cell phenotypic switching in atherosclerosis. Cardiovasc Res 2012; 95(2): 156-164. [DOI: 10.1093/cvr/cvs115]

17. Nurnberg ST, Cheng K, Raiesdana A, Kundu R, Miller CL, Kim JB, et al. Coronary Artery Disease Associated Transcription Factor TCF21 Regulates Smooth Muscle Precursor Cells That Contribute to the Fibrous Cap. PLoS Genet 2015; 11(5): e1005155. [DOI: 10.1371/journal. pgen.1005155]

18. Geovanini GR, Libby P. Atherosclerosis and inflammation: overview and updates. Clin Sci (Lond) 2018;132(12):12431252. [DOI:10.1042/CS20180306]

19. Zengin E, Chalajour F, Gehling UM, Ito WD, Treede H, Lauke H, et al. Vascular wall resident progenitor cells: a source for postnatal vasculogenesis. Development 2006; 133(8): 1543-1551. [DOI: 10.1242/dev.02315]

20. Wirka RC, Wagh DA, Paik DT, et al. Atheroprotective roles of smooth muscle cell phenotypic modulation and the TCF21 disease gene as revealed by single-cell analysis. Nat Med 2019; 25(8): DOI:10.1038/s41591-019-0512-5

21. Shankman LS, Gomez D, Cherepanova OA, Salmon M, Alencar GF, Haskins RM, et al. KLF4-dependent phenotypic modulation of smooth muscle cells has a key role in atherosclerotic plaque pathogenesis. Nat Med 2015; 21(6): 628-637. [DOI: 10.1038/nm.3866]

22. Ghenev PI, Aloe L, Kisheva AR, Singh M, Panayotov P, Fiore M, et al. QUO VADIS, ATHEROGENESIS? Part 1. Smooth muscle cell secretion - how foe becomes friend in the fight against the atherosclerotic plaque. Biomed Rev 2017; 28: 134-138.

23. Chaldakov GN. GEORGE E. PALADE LECTURE. Human body as a multicrine system, with special reference to cell protein secretion: From vascular smooth muscles 
to adipose tissue. Biomed Rev 2016; 27: VIII-XIX.

24. Chaldakov GN, Aloe L, Özturk L, Pancheva R, Fiore $\mathrm{M}$, Yanev $\mathrm{S}$, et al.: Adipokines and myokines (adipomyokines) in diabetes and related cardiometabolic diseases: an (un)expected alliance. In: Gumpeny SR, ed. Advances in Diabetes. Waterstones, 2021 (in press).

25. Töre F, Tonchev AB, Fiore M, Tunçel N, Atanassova P, Aloe L, et al. From Adipose Tissue Protein Secretion to Adipopharmacology of Disease. Immunol Endocr Metab Agents Med Chem 2007; 7: 149-155. [DOI: 10.2174/187152207780363712]

26. Ruddy JM, Ikonomidis JS, Jones JA. Multidimensional Contribution of Matrix Metalloproteinases to Atherosclerotic Plaque Vulnerability: Multiple Mechanisms of Inhibition to Promote Stability. J Vasc Res 2016; 53(1-2): 1-16. [DOI: 10.1159/000446703]

27. Johnson JL. Metalloproteinases in atherosclerosis. Eur J Pharmacol 2017; 816: 93-106. [DOI: 10.1016/j. ejphar.2017.09.007]

28. Wang X, Khalil RA. Matrix Metalloproteinases, Vascular Remodeling, and Vascular Disease. Adv Pharmacol 2018; 81: 241-330. [DOI: 10.1016/bs.apha.2017.08.002]

29. Li T, Li X, Feng Y, Dong G, Wang Y, Yang J. The Role of Matrix Metalloproteinase-9 in Atherosclerotic Plaque Instability. Mediat Inflamm 2020; 2020: 3872367. [DOI: 10.1155/2020/3872367]

30. Olejarz W, Łacheta D, Kubiak-Tomaszewska G. Matrix Metalloproteinases as Biomarkers of Atherosclerotic Plaque Instability. Int J Mol Sci 2020; 21(11). [DOI: 10.3390/ijms21113946]

31. Newby AC. Role of metalloproteinases in plaque rupture. Int J Gerontol 2007; 1(3): 103-111.

32. Rabbani R, Topol EJ. Strategies to achieve coronary arterial plaque stabilization. Cardiovasc Res 1999; 41(2): 402-417. [DOI: 10.1016/s0008-6363(98)00279-x]

33. Masaki M, Kurisaki T, Shirakawa K, Sehara-Fujisawa A. Role of Meltrin $\alpha$ (ADAM12) in Obesity Induced by High-Fat Diet. Endocrinology 2005; 146: 1752-1763. [DOI: 10.1210/en.2004-1082]

34. Salter RC, Ashlin TG, Kwan AP, Ramji DP. ADAMTS proteases: key roles in atherosclerosis? J Mol Med (Berl) 2010; 88(12): 1203-1211. [DOI: 10.1007/s00109-0100654-x]

35. Santamaria S, de Groot R. ADAMTS proteases in cardiovascular physiology and disease. Open Biol 2020; 10(12): 200333. [DOI: 10.1098/rsob.200333]
36. Dong H, Du T, Premaratne S, Zhao CX, Tian Q, Li Y, et al. Relationship between ADAMTS4 and carotid atherosclerotic plaque vulnerability in humans. J Vasc Surg 2018; 67(4): 1120-1126. [DOI: 10.1016/j.jvs.2017.08.075]

37. Zeng T, Gan J, Liu Y, Shi L, Lu Z, Xue Y, et al. ADAMTS-5 Decreases in Aortas and Plasma From Aortic Dissection Patients and Alleviates Angiotensin II-Induced Smooth Muscle-Cell Apoptosis. Front Cardiovasc Med 2020; 7(136): 1-11. [DOI: 10.3389/fcvm.2020.00136]

38. Molloy KJ, Thompson MM, Jones JL, Schwalbe EC, Bell PR, Naylor AR, et al. Unstable carotid plaques exhibit raised matrix metalloproteinase- 8 activity. Circulation 2004; 110(3): 337-343. [DOI: 10.1161/01. CIR.0000135588.65188.14]

39. Newby AC. Metalloproteinases and vulnerable atherosclerotic plaques. Trends Cardiovasc Med 2007; 17(8): 253-258. [DOI: 10.1016/j.tcm.2007.09.001]

40. Silvestre-Roig C, de Winther MP, Weber C, Daemen MJ, Lutgens E, Soehnlein O. Atherosclerotic plaque destabilization: mechanisms, models, and therapeutic strategies. Circ Res 2014; 114(1): 214-226. [DOI: 10.1161/CIRCRESAHA.114.302355]

41. Takata K, Imaizumi S, Zhang B, Miura S-I, Saku K. Stabilization of high-risk plaques. Cardiovasc DiagnTher 2016; 6(4): 304-321. [DOI: 10.21037/cdt.2015.10.03]

42. Ylä-Herttuala S, Bentzon JF, Daemen M, Falk E, GarciaGarcia HM, Herrmann J, et al. Stabilization of atherosclerotic plaques: an update. Eur Heart $J$ 2013; 34(42): 3251-3258. [DOI: 10.1093/eurheartj/eht301]

43. Xiong W, Knispel RA, Dietz HC, Ramirez F, Baxter BT. Doxycycline delays aneurysm rupture in a mouse model of Marfan syndrome. J Vasc Surg 2008; 47(1): 166-172. [DOI: 10.1016/j.jvs.2007.09.016]

44. Dong M, Zhong L, Chen WQ, Ji XP, Zhang M, Zhao YX, et al. Doxycycline stabilizes vulnerable plaque via inhibiting matrix metalloproteinases and attenuating inflammation in rabbits. PloS one 2012; 7(6): e39695-e39695. [DOI: 10.1371/journal.pone.0039695]

45. Suzuki J-i, Ogawa M, Hishikari K, Watanabe R, Takayama $\mathrm{K}$, Hirata Y, et al. Novel Effects of Macrolide Antibiotics on Cardiovascular Diseases. Cardiovasc Ther 2012; 30(6): 301-307. [DOI: 10.1111/j.1755-5922.2011.00303.x]

46. Yakala GK, Cabrera-Fuentes HA, Crespo-Avilan GE, Rattanasopa C, Burlacu A, George BL, et al. FURIN Inhibition Reduces Vascular Remodeling and Atherosclerotic Lesion Progression in Mice. Arteriosc Thromb 
Vasc Biol 2019; 39(3): 387-401. [DOI: 10.1161/ATVBAHA.118.311903]

47. Schulz R, Schlüter KD. PCSK9 targets important for lipid metabolism. Clin Res Cardiol Suppl 2017; 12(Suppl 1): 2-11. [DOI: 10.1007/s11789-017-0085-0]

48. Wang X, Berry E, Hernandez-Anzaldo S, Sun D, Adijiang A, Li L, et al. MMP-2 inhibits PCSK9-induced degradation of the LDL receptor in Hepa1-c1c7 cells. FEBS Lett 2015; 589(4): 490-496. [DOI: 10.1016/j.febslet.2015.01.007]

49. Johnson J, Devel L, Czarny B, George S, Jackson C, Rogakos V, et al. A Selective Matrix Metalloproteinase-12 Inhibitor Retards Atherosclerotic Plaque Development in Apolipoprotein E-Knockout Mice. Arterioscl Thromb Vasc Biol 2011; 31(3): 528-535. [DOI: 10.1161/ATVBAHA.110.219147]

50. Wang C, Jin R, Zhu X, Yan J, Li G. Function of CD147 in atherosclerosis and atherothrombosis. J Cardiovasc Transl Res 2015; 8(1): 59-66. [DOI: 10.1007/s12265-015-96086]

51. Bian H, Zheng Z-H, Wei D, Zhang Z, Kang W-Z, Hao C-Q, et al. Meplazumab treats COVID-19 pneumonia: an open-labelled, concurrent controlled add-on clinical trial. BioRxiv 2020. [DOI: 10.1101/2020.03.21.20040691]

52. Wang M, Zhang J, Spinetti G, Jiang LQ, Monticone R, Zhao D, et al. Angiotensin II activates matrix metalloproteinase type II and mimics age-associated carotid arterial remodeling in young rats. Am J Pathol 2005; 167(5): 1429-1442. [DOI: 10.1016/s0002-9440(10)61229-1]

53. Guo Y-S, Wu Z-G, Yang J-K, Chen X-J. Impact of losartan and angiotensin II on the expression of matrix metalloproteinase- 9 and tissue inhibitor of metalloproteinase-1 in rat vascular smooth muscle cells. Mol Med Rep 2015; 11(3): 1587-1594. [DOI: 10.3892/mmr.2014.2952]

54. Hopps E, Lo Presti R, Caimi G. Matrix Metalloproteases in Arterial Hypertension and their Trend after Antihypertensive Treatment. Kidney Blood Press Res 2017; 42(2): 347-357. [DOI: 10.1159/000477785]

55. Izidoro-Toledo TC, Guimaraes DA, Belo VA, Gerlach RF, Tanus-Santos JE. Effects of statins on matrix metalloproteinases and their endogenous inhibitors in human endothelial cells. Naunyn Schmiedebergs Arch Pharmacol 2011; 383(6): 547-554. [DOI: 10.1007/s00210-011-0623$0]$

56. Marx N, Froehlich J, Siam L, Ittner J, Wierse G, Schmidt A, et al. Antidiabetic PPAR gamma-activator rosiglitazone reduces MMP-9 serum levels in type 2 diabetic patients with coronary artery disease. Arterioscler Thromb Vasc Biol 2003; 23: 283-288.

57. Game BA, Maldonado A, He L, Huang Y. Pioglitazone inhibits MMP-1 expression in vascular smooth muscle cells through a mitogen-activated protein kinase-independent mechanism. Atherosclerosis 2005; 178(2): 249-256. [DOI: 10.1016/j.atherosclerosis.2004.09.012]

58. Kanai K-I, Asano K, Hisamitsu T, Suzaki H. Suppression of matrix metalloproteinase-9 production from neutrophils by a macrolide antibiotic, roxithromycin, in vitro. Mediat Inflamm 2004; 13(5-6): 313-319. [DOI: 10.1080/09629350400008810]

59. Chen P-Y, Qin L, Li G, Tellides G, Simons M. Smooth muscle FGF/TGF $\beta$ cross talk regulates atherosclerosis progression. EMBO Mol Med 2016; 8(7): 712-728. [DOI: 10.15252/emmm.201506181]

60. Li J, Xue H, Li T, Chu X, Xin D, Xiong Y, et al. Exosomes derived from mesenchymal stem cells attenuate the progression of atherosclerosis in ApoE(-/-) mice via miR-let7 mediated infiltration and polarization of M2 macrophage. Biochem Biophys Res Commun 2019; 510(4): 565-572. [DOI: 10.1016/j.bbrc.2019.02.005]

61. Di Gregoli K, Mohamad Anuar NN, Bianco R, White SJ, Newby AC, George SJ, et al. MicroRNA-181b Controls Atherosclerosis and Aneurysms Through Regulation of TIMP-3 and Elastin. Circ Res 2017; 120(1): 49-65. [DOI: 10.1161/circresaha.116.309321]

62. Cecconi A, Vilchez-Tschischke JP, Mateo J, SanchezGonzalez J, España S, et al. Effects of Colchicine on Atherosclerotic Plaque Stabilization: a Multimodality Imaging Study in an Animal Model. J Cardiovasc Transl Res [DOI:10.1007/s12265-020-09974-7]

63. Xie Y, Martin KA. TCF21: Flipping the phenotypic switch in SMC. Circ Res 2020;126(4):530-532. [DOI: 10.1161/ CIRCRESAHA.120.316533]

64. Shafi O. Switching of vascular cells towards atherogenesis, and other factors contributing to atherosclerosis: a systematic review. Thromb J 2020;18:28

65. Kattoor AJ, Pothineni NVK, Palagiri D, Mehta JL. Oxidative Stress in Atherosclerosis. Curr Atheroscler Rep 2017;19(11):42. [DOI: 10.1007/s11883-017-0678-6]

66. Chaldakov GN. Anti-inflammatory drugs and ischemic heart disease: New considerations (A cell biologist's proposal to cardiologists). J Am Coll Cardiol 1991;17(6):1445-1447. 